\title{
THE CEREBELLUM, A SMALL BRAIN WITH A BIG OUTCOME
}

\section{S. Baader}

Anatomy and Cell Biology, Institute of Anatomy, Bonn, Germany

The simple three-layered cortex, the well-defined connectivity, the anatomical accessibility as well as genetical tools available to manipulate cell-type specific gene expression made the cerebellum a favourable model system, in which basic mechanisms of central nervous system development can be investigated. The fundamental knowledge about cerebellar structure and development improved our understanding of clinical symptoms. The definition of compartments, migrational paths of neurons and neuronal differentiation are topics which will be discussed in this context. As the cerebellum develops out of the mid-hindbrain junction, it is clearly divided into a vermal and hemispheric region. The mechanistic basis of the setup and maintenance of this division is of major importance, not only for the spatially organized connectivity of the cerebellum to afferent and efferent projection fields, but also for answering questions about the origin of medulloblastomas, the regional differences in the sensitivity towards neurodegeneration or midline defects. The cells of the cerebellum migrate along distinct paths either in a radial or transverse direction. Mechanisms guiding neural cells to their final destination are considered causative for many neuronal heterotopias. And finally, the differentiation of neurons is essential for proper neurotransmission and neuronal communication. The Purkinje cells provide a historical, but still suitable attractive and often used model cell to investigate neuronal differentiation and maturation. Our knowledge about neuronal differentiation helped to understand neuropsychiatric disorders of adult, but also of newborns and tottlers. 